INPLASY

PROTOCOL

To cite: Xiao et al. Correlation between psychosocial stress factors and the incidence of allergic rhinitis in adults: systematic review and metaanalysis. Inplasy protocol

202170025. doi:

10.37766/inplasy2021.7.0025

Received: 09 July 2021

Published: 09 July 2021

Corresponding author:

Yao Xiao

1399073323@qq.com

Author Affiliation:

Jiangxi University of Chinese

Medicine

Support: Unfunded.

Review Stage at time of this submission: The review has not yet started.

Conflicts of interest:

None declared.

\section{Correlation between psychosocial stress factors and the incidence of allergic rhinitis in adults: systematic review and meta-analysis}

\author{
Xiao, Y1; Xiong, J2; Zeng, Y3; Li, J4.
}

Review question / Objective: To investigate the influence of psychosocial stress factors on allergic rhinitis. Do adult psychosocial stress and adverse life events influence the occurrence of allergic rhinitis? Whether the influence of adult psychosocial stress is influenced by the type of stress exposure or the adverse life Event, age at exposure, age at diagnosis/outcome assessment or Gender?

Condition being studied: As a global public health problem, the prevalence of allergic rhinitis (AR) is still on the rise His whole world. There are a number of studies that have found a strong correlation between psychosocial stress factors The relationship between. Therefore, studies on the correlation between psychosocial stress factors and the incidence of AR are Necessary and valuable, but no relevant meta-analysis

INPLASY registration number: This protocol was registered with the International Platform of Registered Systematic Review and Meta-Analysis Protocols (INPLASY) on 09 July 2021 and was last updated on 09 July 2021 (registration number INPLASY202170025).

\section{INTRODUCTION}

Review question / Objective: To investigate the influence of psychosocial stress factors on allergic rhinitis. Do adult psychosocial stress and adverse life events influence the occurrence of allergic rhinitis? Whether the influence of adult psychosocial stress is influenced by the type of stress exposure or the adverse life Event, age at exposure, age at diagnosis/outcome assessment or Gender?

Condition being studied: As a global public health problem, the prevalence of allergic rhinitis (AR) is still on the rise His whole 
world. There are a number of studies that have found a strong correlation between psychosocial stress factors The relationship between. Therefore, studies on the correlation between psychosocial stress factors and the incidence of AR are Necessary and valuable, but no relevant meta-analysis

\section{METHODS}

Participant or population: Inclusion criteria AR patients according to accepted diagnostic criteria (regardless of age or sex) are included; - Quantitative data for estimating the risk of allergic rhinitis symptoms based on indicators of adverse psychosocial stressors (e.g., socioeconomic status, post-traumatic stress disorder, work stress, marital status, VE, combined stressors) in allergic rhinitis are reported. - It uses criteria to measure psychosocial factors related to stress, including evidence-based scales such as the Work Demand-Control-Social Support Scale and the Effort-Reward Disequilibrium Scale. - It provides analysis results adjusted for age and sex; We contacted the study authors who reported unadjusted results and included new adjusted data if provided. - The sample included at least $50 \%$ of individuals over 18 years of age who were identified as having allergic rhinitis. If a younger population is sampled, include the study if the study proposes a stratification of older people over 65 years of age.

Intervention: Exposure to psychosocial stress variables such as racism, perceived stress, discrimination, depression, anxiety, poverty). (Short and Long Term)

Comparator: Not applicable.

Study designs to be included: Types of study to be includedinclusion criteria: Study design of case-control, crosssectional or cohort study will be included.Exclusion criteria: reviews, editorials, commentaries, letters, methodological papers or experimental studies.
Eligibility criteria: Inclusion criteria: 1) Results The sample size and association (COR) between psychosocial stressors and the onset of AR were reported. 2) Risk is reported, such as relative risk (RR) and associated $95 \%$ confidence intervals $(\mathrm{Cl})$ or other data to estimate variance or accuracy (i.e., standard deviation or standard error); 3) The risk assessment was adjusted for potential confounders or other forms of standardization (if applicable).Exclusion criteria: 1) For duplicate literatures, only literatures with good quality and complete information should be retained, and other literatures should be deleted. 2) Unable to extract data for statistical analysis; 3) Psychosocial stress is caused by a single extreme event (e.g., poverty). 4) Poor literature quality or statistical errors; 5) reviewed; Comments. Letters.

Information sources: We will search PubMed(MEDLINE), EMBASE, Cochrane Library, Web of Science, China National Knowledge Infrastructure(CNKI), VIP, Sinomed and Wanfang to identify all published studies, There will be no language restrictions. (The deadline is July $6,2021)$.

Main outcome(s): Psychosocial stress factSecondary outcomes will include any measures of disease severity or impact, including: frequency of allergic rhinitis episodes, use of allergic rhinitis medications, hospitalization for allergic rhinitis, self-reported or physiciandiagnosed definition of allergic rhinitis, and measures of health-related quality of life (HRQOL). Psychosocial stress factors related to the onset of allergic rhinitis.

\section{Additional outcome(s): Not applicable.}

Quality assessment / Risk of bias analysis: Every work group has three members. Two of them will independently screen the studies and extract the relative data, following pre-defined selection criteria. The third member will decide the unsure study whether included or excluded and check the data extraction table. For the selected 
studies, information such as the first author, publication date, title, source, region, sample size and unit, psychosocial stress factors and their correlation coefficients with AR were extracted and input into Microsoft Excel.

Strategy of data synthesis: Data will be analyzed using Excel and STATA13.1. When heterogeneity occurs across studies, if the $p$-value was $<0.05$, a random effect model would be selected;if not, a fixed-effects model.Summary effect sizes will be calculated as RR(or AR), with $95 \%$ credible intervals (Crls).The $Q$ and $I^{2}$ statistics will be used to test for heterogeneity between included studies.Sensitivity analysis was performed to investigate the influence of a single study on the overall meta-analysis estimate.

Subgroup analysis: Subgroup analyses were based on the different continent, country or district (depands on the actually extracted data).

Sensitivity analysis: To assess the influence of each individual study, leave-one-out sensitivity analysis was performed iteratively by removing one study at a time to confirm that the findings were not influenced by any single study.

Language: English.

Country(ies) involved: China.

Keywords: Allergic rhinitis, Mental Disorders; Risk; Stress; Psychological; Mental Health.

Contributions of each author:

Author 1 - Yao Xiao - The author drafted and improved the manuscript.

Email: 1399073323@qq.com

Author 2 - Jun Xiong - Revise this protocol; search strategy.

Email: xiongjun196071@163.com

Author 3 - Yuntao Zeng - Data collection; analysis of results.

Email: 1451911996@qq.com

Author 4 - Jun Li - Data collection.

Email: 1030083634@qq.com 